\title{
miR-125/CDK2 axis in cochlear progenitor cell proliferation
}

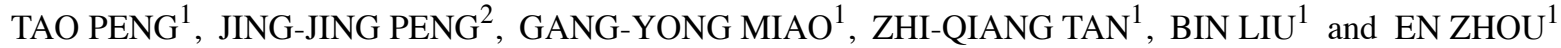 \\ ${ }^{1}$ Department of Otolaryngology and Head and Neck Surgery, Hunan Provincial People's Hospital, \\ First Affiliated Hospital of Hunan Normal University, Changsha, Hunan 410007; ${ }^{2}$ Department of Obstetrics and \\ Gynecology, Changsha Maternal and Child Health Care Hospital, Changsha, Hunan 410005, P.R. China
}

Received July 13, 2020; Accepted October 16, 2020

DOI: $10.3892 / \mathrm{mmr} .2020 .11741$

\begin{abstract}
Hearing loss ranks fourth among the principal causes of disability worldwide, and manipulation of progenitor cells may be a key strategy for hair cell regeneration. The present study investigated the role and mechanism of miR-125 on the proliferation of cochlear progenitor cells (CPCs). CPCs were isolated from the cochleae of neonatal rats, and their morphology was observed. Furthermore, the differentiation ability of CPCs was determined by assessing the expression of 5-bromodeoxyuridine (BrdU), nestin and myosin VII by immunofluorescence. The expression levels of miR-125 and cyclin-dependent kinase 2 (CDK2) as well as the cell proliferation of CPCs were assessed. In addition, following gain- and loss-of-function assays, the cell cycle was examined by flow cytometry, and the expression levels of miR-125, CDK2, proliferating cell nuclear antigen (PCNA) and nestin were determined by reverse transcription-quantitative PCR and western blotting. The binding sites between miR-125 and CDK2 were predicted by TargetScan and identified by the dual luciferase reporter assay. The results demonstrated that different types of progenitor spheres were observed from CPCs with positive expression of BrdU, nestin and myosin VII. Following in vitro incubation for 2, 4 and 7 days, the spheres were enlarged, and CPC proliferation gradually increased and reached a plateau after further incubation for 3 days. Furthermore, the expression levels of nestin and PCNA in CPCs increased and then decreased during in vitro incubation for 2, 4 and 7 days. Following this incubation, the expression levels of miR-125 in CPCs decreased; thereafter, its expression increased, and the expression pattern was different from that of CDK2. In addition, miR-125 overexpression in CPCs decreased the expression of CDK2 and the number of cells in the $\mathrm{S}$ phase. Different expression patterns were found
\end{abstract}

Correspondence to: Dr Tao Peng, Department of Otolaryngology and Head and Neck Surgery, Hunan Provincial People's Hospital, First Affiliated Hospital of Hunan Normal University, 61 West Jiefang Road, Furong, Changsha, Hunan 410007, P.R. China

E-mail:pt7240@163.com

Key words: miR-125, cochlear progenitor cell, cyclin-dependent kinase 2, bromodeoxyuridine, nestin in CPCs in response to the miR-125 knockdown. In addition, miR-125 directly targeted CDK2. Simultaneous knockdown of miR-125 and CDK2 enhanced CPC proliferation compared with CDK2 knockdown alone. Taken together, the findings from the present study suggested that miR-125 may inhibit CPC proliferation by downregulating CDK2. The present study may provide a novel therapeutic direction for treatment of hearing loss.

\section{Introduction}

Hearing loss ranks fourth among the principal causes of disability worldwide and leads to impaired communication, social isolation and reduced quality of life (1). Hearing loss is a common sensory disorder that results from genetic and environmental factors, including genetic mutations, ototoxic drugs, noise exposure and ageing (2). These physicochemical or pathological factors could induce the damage or loss of human inner ear hair cells (3). Currently, the most common therapy for sensorineural hearing loss is hearing rehabilitation with hearing devices; however, the sound quality perceived is not as good as with the original cochlea (4). Sensory hair cells are located in the organ of Corti and act as mechanosensory cells (5). Mammalian auditory hair cells cannot self-regenerate; therefore, the hearing loss caused by hair cell loss is permanent (6). Regeneration of cochlear hair cells has been considered a promising treatment approach for noise-induced sensorineural hearing loss and age-related hearing loss $(7,8)$. It was demonstrated that cochlear progenitor cells (CPCs) can differentiate into either sensory hair cells or supporting cells, whose number is the determinant factor of the final length of the cochlea (9), indicating that manipulation of progenitor cells could be considered as a key point for hair cell regeneration. Further investigation on the mechanism of CPC proliferation is therefore required.

Any dysregulation of microRNA (miRNA) in the cochlea is likely to damage the structure of the auditory system and cause hearing loss (10). For instance, it has been reported that an increase in miR-34a level is associated with increased hearing threshold and a larger loss of hair cells in the cochlea (11). However, the impact of dysregulated miRNAs on the biological functions of CPCs remains unclear. In retinal progenitors, miR-125 has been reported to be expressed, but its expression is altered over the developmental period (12). Furthermore, miR-125 significantly suppresses the proliferation of cardiac 
progenitor cells during hypoxia (13). However, the expression and function of miR-125 in CPCs remain unclear. It has been reported that cyclin-dependent kinase 2 (CDK2) activity could determine whether eukaryotic cells enter the next cell cycle or enter a transient G0-like state at the end of mitosis (14), suggesting the pivotal role of CDK2 in cell biological functions. Further investigating the effects of the miR-125/CDK2 axis on the biological process of CPCs is therefore required.

The present study aimed to identify the expression levels and function of miR-125 in CPCs and to elucidate the potential mechanism by which miR-125 regulates the proliferation of CPCs. Identifying the role and underlying mechanism of miR-125 in CPC proliferation may provide novel insights towards the regeneration of hair cells and treatment of hearing loss.

\section{Materials and methods}

Ethical statement. Animal experiments were approved by the local Ethics Committee of Hunan Provincial People's Hospital. All animals were cared for according to the Guide for the Care and Use of Laboratory Animals [National Institutes of Health (NIH); version 8, 2011].

Animals. Neonatal rats (0-3 days old) were purchased from Shanghai Model Organisms Center, Inc. and housed under specific pathogen-free conditions at humidity of $60-65 \%$.

Isolation and culture of CPCs. After being intraperitoneally anaesthetized by $2 \%$ pentobarbital sodium $(40 \mathrm{mg} / \mathrm{kg})$, the neonatal rats were sterilized in $75 \%$ ethanol and sacrificed by decapitation. The bilateral temporal bones were obtained and then placed in precooled $\left(4^{\circ} \mathrm{C}\right){ }^{\circ}$ normal saline. The cochlear and spiral ligaments were removed by microdissection. After being washed with Hank's balanced salt solution (HBSS; HyClone; GE Healthcare Life Sciences) twice, cochlear tissues were cut into pieces $\left(0.5 \mathrm{~mm}^{3}\right)$. Then, the tissues were digested by $0.125 \%$ tryptase at $37^{\circ} \mathrm{C}$ for $20 \mathrm{~min}$, during which the tissues were flipped every 5 min using a fire-polished Pasteur pipet. Subsequently, $10 \%$ foetal calf serum (FCS, HyClone; GE Healthcare Life Sciences) was added for $5 \mathrm{~min}$ to terminate the digestion at room temperature. Eventually, samples were centrifuged at $111 \mathrm{x} \mathrm{g}$ for $5 \mathrm{~min}$ at room temperature. The supernatant was discarded and the pellets were washed with HBSS twice. DMEM/F12 (HyClone; GE Healthcare Life Sciences) containing B27 (1:50) and N2 (1:100; both Sigma-Aldrich; Merck KGaA) was used to resuspend cells that were filtered using a 100-mesh copper net (pore size, $70 \mu \mathrm{m}$ ). A single cell suspension was prepared using DMEM/F12 supplemented with epidermal growth factor $(20 \mathrm{ng} / \mathrm{ml})$, basic fibroblast growth factor $(20 \mathrm{ng} / \mathrm{ml})$ and penicillin $(100 \mathrm{U} / \mathrm{ml})$ (PeproTech China). Living cells $\left(5 \times 10^{5} \mathrm{ml}\right)$ were placed in 24-well plates and cultured at $37^{\circ} \mathrm{C}$ in a humidified incubator containing $5 \% \mathrm{CO}_{2}$. The culture medium was replaced every other day. Cells were passaged every 5 days at a density of $5 \times 10^{5} / \mathrm{ml}$. Cells used for in vitro differentiation were subjected to suspension culture at $37^{\circ} \mathrm{C}$ for 3 days. Then, the culture medium was replaced with the aforementioned culture medium containing $10 \%$ FCS. Afterwards, the cells were further cultured at $37^{\circ} \mathrm{C}$ for 12 days to induce differentiation.
In vitro differentiation of rat CPCs. The 3rd generation of CPCs was cultured for 4 days, then the CPCs were centrifuged at $88 \times \mathrm{g}$ for $10 \mathrm{~min}$ at room temperature. Supernatant was removed and cells were resuspended in DMEM/F12 containing $10 \%$ FCS. Cells $\left(1 \times 10^{4}\right)$ were incubated in culture dishes that contained polylysine-encased coverslips at $37^{\circ} \mathrm{C}$ with $5 \% \mathrm{CO}_{2}$ for $12 \mathrm{~h}$. Once cells had adhered, the supernatant was discarded and the medium was replaced. Half of the medium was replaced every other day until the seventh day. The medium consisted of DMEM/F12 solution, $100 \mathrm{U} / \mathrm{ml}$ penicillin-streptomycin, 2\% B27 and 2\% N2.

Cell proliferation evaluation by MTT assay. Cells (1x10 $/ \mathrm{ml})$ in different growth phases were seeded in a 96 -well plate (100 $\mu \mathrm{l} /$ well) and incubated at $37^{\circ} \mathrm{C}$ with $5 \% \mathrm{CO}_{2}$. Then, cells were incubated with $10 \mu \mathrm{l}$ of $5 \mathrm{mg} / \mathrm{ml}$ MTT (Beyotime Institute of Biotechnology) for $4 \mathrm{~h}$ at $37^{\circ} \mathrm{C}$. Subsequently, the MTT solution was discarded and $20 \%$ sodium dodecyl sulphate $\left(100 \mu \mathrm{l} /\right.$ well) was added to the cells for $4 \mathrm{~h}$ at $37^{\circ} \mathrm{C}$ with $5 \% \mathrm{CO}_{2}$ to terminate the reaction. The absorbance was read at $570 \mathrm{~nm}$ using a microplate reader (SpectraMax 190; Molecular Devices, LLC).

Cell transfection. After incubation for 2 days, CPCs were transfected with miR-125 mimic (50 nM), miR-125 inhibitor $(50 \mathrm{nM})$, small interfering (si)-CDK2 $(2 \mu \mathrm{g})$ or the corresponding negative controls (NC mimic, NC inhibitor or si-NC) or were co-transfected with miR-125 inhibitor + si-CDK2 (all Shanghai GenePharma Co., Ltd.) using Lipofectamine 2000 (Thermo Fisher Scientific, Inc.). The sequences were as follows: $\mathrm{miR}-125 \mathrm{mimic}$, forward 5'-ACA AGUCAGGUUCUUGGGACCU-3', reverse 3'-UGUUCA GUCCAAGAACCCUGGA-5'; mimic NC, forward 5'-ACA CGUCAGCAUUAACUCCUUG-3', reverse 3'-UGUGCA GUCGUAAUUGAGGAAC-5'; miR-125 inhibitor 5'-UGU UCAGUCCAAGAACCCUGGA-3'; inhibitor NC, 5'-ACU GCCAUCUAUCUCGGAACGA-3'; si-CDK2, GGTGTACCC AGTACTGCCA; si-NC GACTTCATAAGGCGATGC. Cell transfection was performed for $48 \mathrm{~h}$ at room temperature, after which cells were cultured at $37^{\circ} \mathrm{C}$ for $48 \mathrm{~h}$ before subsequent experiments.

Immunofluorescence. Cells were collected and resuspended in culture medium containing $10 \%$ FCS. Cells were then seeded onto polylysine-treated coverslips at a density of $1 \times 10^{5}$ and incubated at $5 \% \mathrm{CO}_{2}$ and $37^{\circ} \mathrm{C}$. Following incubation for $24 \mathrm{~h}$, the cells used for identification of CPCs and differentiation were fixed with $4 \%$ paraformaldehyde for $20 \mathrm{~min}$ at room temperature and washed with PBS three times. Subsequently, cells were incubated with $1 \%$ Triton X-100 on ice for $5 \mathrm{~min}$, washed with PBS three times and blocked with $5 \%$ bovine serum albumin (cat. no. ST025; Beyotime Institute of Biotechnology) diluted in PBS. The blocking buffer was discarded and cells were incubated with primary antibodies against nestin (1:100; cat. no. MAB353; EMD Millipore), BrdU (1:500; cat. no. MAB4072; EMD Millipore) and myosin VIIA (1:100; cat. no. Ab150386; Abcam) at $4^{\circ} \mathrm{C}$ overnight. Subsequently, cells were washed with PBS three times and incubated with Cy3-labelled secondary antibody $\mathrm{IgG}$ (1:100; Sigma-Aldrich; Merck KGaA) at $37^{\circ} \mathrm{C}$ for $30 \mathrm{~min}$. 
The nuclei were stained with DAPI $(5 \mu \mathrm{g} / \mathrm{ml}$; cat. no. C1002; Beyotime Institute of Biotechnology) at room temperature for 5 min. After being washed with PBS three times, cells were visualized and imaged under a fluorescence microscope (magnification, x400; Olympus BX51; Olympus Corporation).

Cell cycle analysis. Cells were washed with ice-cold PBS three times and centrifuged at $1,000 \mathrm{x} g$ at room temperature for $5 \mathrm{~min}$. Cells were suspended in ice-cold PBS and fixed in absolute ethanol for $30 \mathrm{~min}$ at $4^{\circ} \mathrm{C}$. The ethanol was discarded after incubation and cells were washed with PBS to remove the residual ethanol. Subsequently, cells were resuspended in PBS with $3 \mu 1$ RNAse (cat. no. ST578; Beyotime Institute of Biotechnology) and incubated at $37^{\circ} \mathrm{C}$ for $30 \mathrm{~min}$. Eventually, cells were stained with propidium iodide (PI; $50 \mu \mathrm{g} / \mathrm{ml}$; Sigma-Aldrich; Merck KGaA) for $30 \mathrm{~min}$ at $37^{\circ} \mathrm{C}$. Cell cycle distribution was detected by flow cytometry and data were analyzed using CellQuest Pro software version 5.1 (FACSCalibur; Becton, Dickinson and Company).

Reverse transcription quantitative (RT-q) PCR. CPCs were lysed in $1 \mathrm{ml} \mathrm{TRIzol}{ }^{\circledR}$ (Thermo Fisher Scientific, Inc.), and total RNA was extracted according to the manufacturer's instructions. Following RNA quantification, cDNA was obtained by reverse transcription using BeyoRT ${ }^{\mathrm{TM}} \mathrm{II}$ and cDNA synthesis kit (cat. no. D7168M; Beyotime Institute of Biotechnology), and then subjected to RT-qPCR using a Quanti Fast SYBR ${ }^{\circledR}$ Green PCR kit (Qiagen, Inc.). The procedures were performed as follows: 40 cycles of pre-degradation at $95^{\circ} \mathrm{C}$ for $2 \mathrm{~min}$, degradation at $95^{\circ} \mathrm{C}$ for $10 \mathrm{sec}$, annealing at $60^{\circ} \mathrm{C}$ for $40 \mathrm{sec}$ and extension at $72^{\circ} \mathrm{C}$ for $20 \mathrm{sec}$. Each experiment was repeated three times. The relative expression levels were normalized to endogenous controls GAPDH or U6 (for endogenous normalization for miRNA) and were expressed as $2^{-\Delta \Delta C q}(15)$. The sequences of the primers used are presented in Table $\mathrm{I}$.

Western blotting. CPCs were lysed using RIPA lysis buffer (Beyotime Institute of Biotechnology) on ice and the proteins were quantified using bicinchoninic acid (Beyotime Institute of Biotechnology). Proteins (50 $\mu \mathrm{g} / \mathrm{lane})$ were separated by $10 \%$ SDS-PAGE and transferred onto PVDF membranes. Subsequently, the membranes were blocked with Tris buffer containing 5\% non-fat milk at room temperature for $1 \mathrm{~h}$ and incubated with primary antibodies against GAPDH (1:10,000; cat. no. ab181602; Abcam), CDK2 (1:1,000; cat. no. ab32147; Abcam), proliferating cell nuclear antigen (PCNA; 1:1,000; ab92552; Abcam), nestin (1:1,000; cat. no. ab6142; Abcam) and $\beta$-catenin (1:5,000; cat. no. ab32572; Abcam) at $4^{\circ} \mathrm{C}$ overnight. Membranes were washed with PBST (10\% Tween-20) three times and were incubated with goat anti-rabbit $\operatorname{IgG}(1: 5,000$; cat. no. CW0103S; CoWin Biosciences) or goat anti-rat IgG (1:2,000; cat. no. ab205719; Abcam) secondary antibodies at room temperature for $30 \mathrm{~min}$. Membranes were washed with PBST four times and enhanced chemiluminescence reagent (cat. no. CW0049S; CoWin Biosciences) was used to detect the signal on the membrane using a chemiluminescence imaging system (GE Healthcare). ImageJ software (version 1.46; NIH) was used to analyze protein bands.
Table I. Sequences of the primers used for reverse transcription quantitative PCR.

\begin{tabular}{ll}
\hline Name & \multicolumn{1}{c}{ Primer sequence $\left(5^{\prime} \rightarrow 3^{\prime}\right)$} \\
\hline miR-125 & \\
Forward & TCCAGGGTTCTTGGAC \\
Reverse & GCAGGGTCCGAGGTATTC \\
CDK2 & \\
Forward & TGCCCTTTCACTGCCTATGG \\
Reverse & GAGGAAAGCCAAGACCCACA \\
PCNA & \\
Forward & CTCCTCATCCTTGCGTCCTCATAT \\
Reverse & GAGGCACTTGGCAATGTATTCGATAT \\
Nestin & \\
Forward & ATCTACACATACACGGGTTCCA \\
Reverse & TTCTTCTTCTCCTCCTCATTCA \\
U6 & \\
Forward & CTCGCTTCGGCAGCACA \\
Reverse & AACGCTTCACGAATTTGCGT \\
GAPDH & \\
Forward & TCTTGTGCAGTGCCAGCCT \\
Reverse & TGAGGTCAATGAAGGGGTCG \\
\hline
\end{tabular}

CDK2, cyclin-dependent kinase 2; PCNA, proliferating cell nuclear antigen.

Dual luciferase reporter assay. TargetScan (targetscan. org/vert_72/) was used to predict the binding sites between miR-125 and CDK2. According to the prediction, wild-type (wt) and mutant (mut)-type sequences of the binding sites between miR-125 and CDK2 were synthesized and cloned into the reporter vectors (pGL3-Basic, Promega Corporation), which were named mut-CDK2 and wt-CDK2. 293T cells (American Type Culture Collection) were co-transfected with mut-CDK2 or wt-CDK2 and miR-125 mimic, miR-125 inhibitor, NC mimic or NC inhibitor (GenePharma, Shanghai, China). OPTI-MEM (49 $\mu \mathrm{l}$ ) was pipetted onto 24 -well plates to dilute $1 \mu 1$ Lipofectamine 2000 reagent (Invitrogen; Thermo Fisher Scientific, Inc.), and the final volume was $50 \mu 1$. After $48 \mathrm{~h}$ transfection, luciferase activity was detected using a Lucifer Reporter analytic system (Promega Corporation) by comparison with Renilla luciferase activity.

Statistical analysis. Statistical analysis was performed using GraphPad Prism 7.0 (GraphPad Software, Inc.). Comparisons between two groups were measured by Student's t-test, whereas comparisons among multiple groups were conducted by one-way analysis of variance followed by Tukey's post hoc test. $\mathrm{P}<0.05$ was considered to indicate a statistically significant difference.

\section{Results}

Evaluation of the CPC in vitro differentiation model. After culture, cells that were isolated from the cochleae of neonatal 

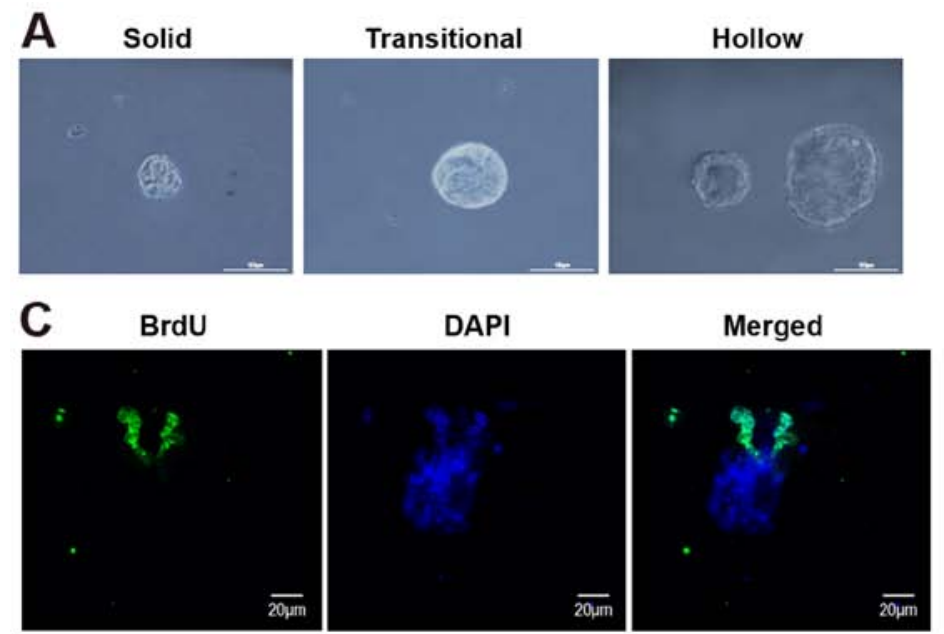
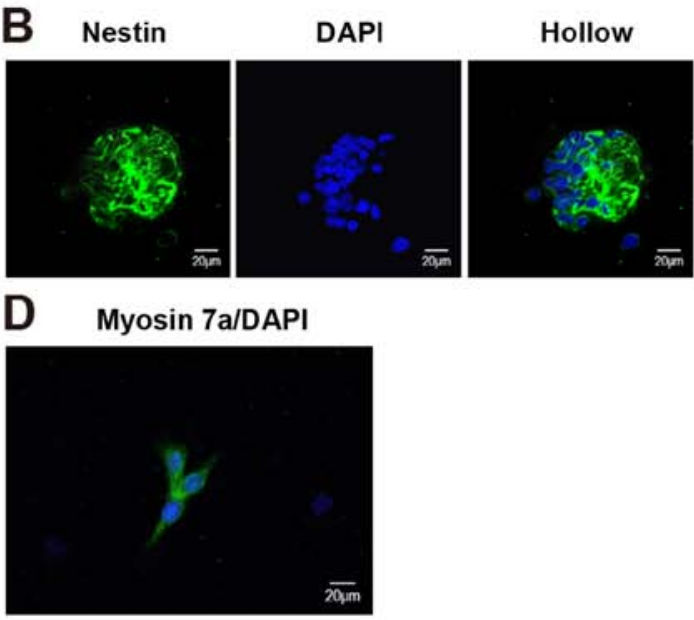

Figure 1. Isolated cells were able to differentiate into hair cells. (A) Cochlear progenitor cells were isolated and cultured in vitro, and the formation of progenitor spheres was observed using an inverted microscope. Magnification, x600; Scale bar, $100 \mu \mathrm{m}$. Immunofluorescence was used to detect cells that were positive for (B) BrdU and (C) nestin. (D) Detection of myosin VIIA expression by immunofluorescence. Magnification, $\mathrm{x} 400$; Scale bar, $20 \mu \mathrm{m}$.

rats formed progenitor spheres with different morphologies, including solid, transitional and hollow spheres (Fig. 1A). Furthermore, a fraction of CPCs was positive for nestin and BrdU. As a marker of neural stem cells, nestin was mainly expressed in the cytoplasm (Fig. 1B), and BrdU, which is a marker for mitosis, was mainly expressed in the nuclei (Fig. 1C). To identify the directional differentiation cell capacity, in vitro directional differentiation was induced for 12 days. Immunofluorescence was used to detect the expression of the hair cell marker myosin VII, and the results suggested that myosin VII was expressed in the isolated cells (Fig. 1D), which confirmed that the isolated cells had the potential to differentiate into hair cells.

Identification of CPC proliferation. The three types of progenitor spheres had different proportions depending on culture durations. For spheres cultured for 2, 4 and 7 days, the size of the progenitor spheres gradually increased in a time-dependent manner (Fig. 2A). During this time, the proportion of solid spheres decreased and the proportion of hollow spheres increased (Fig. 2B). CPCs in different growth phases were subjected to MTT to measure their proliferation abilities. The results demonstrated that CPCs were in the exponential phase after incubation for 1-3 days, and the cells reached a plateau after 3 days of incubation (Fig. 2C; $\mathrm{P}<0.05)$. The results from RT-qPCR demonstrated that the expression levels of nestin and PCNA were significantly increased and decreased after 4 and 7 days, respectively (Fig. 2D; $\mathrm{P}<0.05$ ).

To investigate the role of miR-125 in CPC proliferation, miR-125 expression was detected by RT-qPCR. In the spheres cultured for 4 days, the expression of miR-125 was significantly lower than that in the spheres cultured for 2 days (Fig. 2E; $\mathrm{P}<0.05$ ). Furthermore, in the spheres cultured for 7 days, miR-125 expression was higher than that in the spheres cultured for 4 days (Fig. 2E; $\mathrm{P}<0.01$ ). In addition, results from RT-qPCR demonstrated that CDK2 expression level was significantly increased in spheres cultured for 4 days compared with spheres cultured for 2 days (Fig. $2 \mathrm{~F}$; $\mathrm{P}<0.01$ ). In addition,
CDK2 expression level decreased significantly in the spheres cultured for 7 days compared with spheres cultured for 4 days (Fig. 2F; $\mathrm{P}<0.01$ ). These findings were confirmed by western blotting (Fig. 2G; $\mathrm{P}<0.05$ ).

Inhibitory effect of miR-125 on CPC proliferation. To further explore the effect of miR-125 on CPC proliferation, CPCs cultured for 2 days were transfected with miR-125 mimic or miR-125 inhibitor. As confirmed by RT-qPCR, miR-125 expression was elevated in the miR-125 mimic group compared with the NC mimic group, and the expression of miR-125 was decreased in the miR-125 inhibitor group compared with the NC inhibitor group (Fig. 3A; $\mathrm{P}<0.001$ and $\mathrm{P}<0.01$ ). Furthermore, the expression level of CDK2 was significantly decreased in the miR-125 mimic group compared with the NC mimic group (Fig. 3B; $\mathrm{P}<0.01$ ), and significantly increased in the miR-125 inhibitor group compared with $\mathrm{NC}$ inhibitor group (Fig. 3B; $\mathrm{P}<0.01$ ). These results were confirmed by western blotting (Fig. 3C). These findings suggested that miR-125 could negatively regulate CDK2. Furthermore, the results from RT-qPCR demonstrated that nestin and PCNA were significantly downregulated in the miR-125 mimic group compared with the NC mimic group, and significantly upregulated in the miR-125 inhibitor group compared with the NC inhibitor group (Fig. 3D; $\mathrm{P}<0.01$ ). These data were confirmed by western blotting (Fig. 3E; $\mathrm{P}<0.05$ ). The detection of the cell cycle by flow cytometry demonstrated that miR-125 inhibition increased the number of cells in the $\mathrm{S}$ phase, and that miR-125 overexpression had the opposite effect (Fig. 3F; P<0.05). Taken together, these findings suggested that miR-125 may inhibit CPC proliferation.

miR-125 exerts its regulatory effect on CPCs via CDK2. TargetScan (targetscan.org/vert_72/) results demonstrated that there were binding sites between miR-125 and CDK2 (Fig. 4A). According to these results, wt-CDK2 and mut-CDK2 were constructed, and 293T cells were co-transfected with wt-CDK2 or mut-CDK2 and miR-125 mimic or miR-125 inhibitor. The results from dual luciferase reporter assay 
A

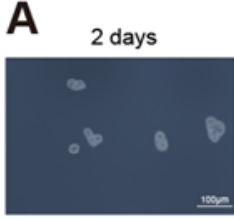

C

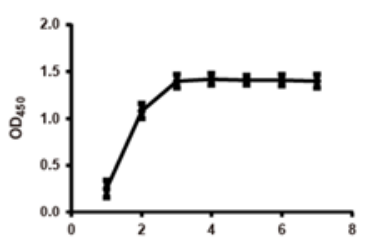

G

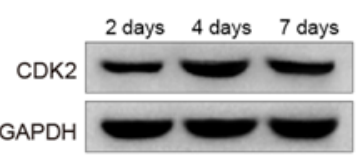

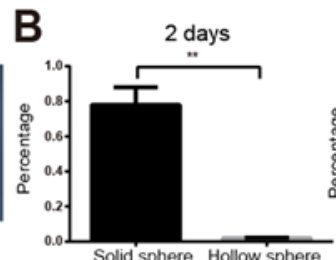

E

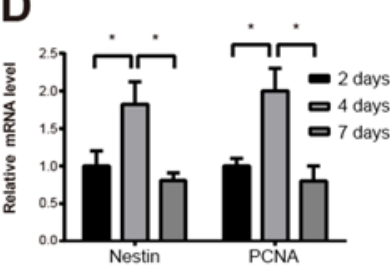

2 days

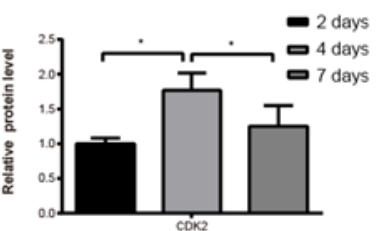

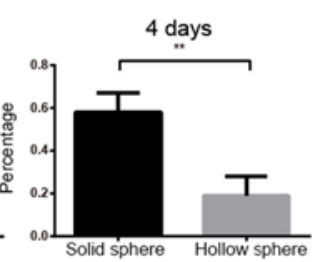
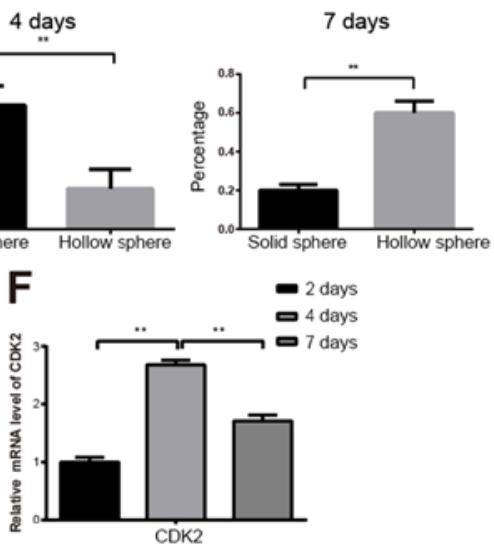

Figure 2. Proliferation ability of CPCs decreases concurrent with increased miR-125 and downregulated CDK2 expression. (A) After in vitro incubation for 2, 4 and 7 days, progenitor spheres were observed using an inverted microscope. Scale bar, $100 \mu \mathrm{m}$. (B) Proportion of different types of progenitor spheres. (C) Proliferation of CPCs assessed by MTT. (D) RT-qPCR analysis of nestin and PCNA. (E) Detection of miR-125 expression by RT-qPCR. CDK2 expression detected by (F) RT-qPCR and (G) western blotting. All data were expressed as the means \pm standard deviation. ${ }^{*} \mathrm{P}<0.05$ and ${ }^{* * *} \mathrm{P}<0.01$. CPCs, cochlear progenitor cells; RT-qPCR, reverse transcription quantitative PCR; CDK2, cyclin-dependent kinase 2; OD, optical density.

A

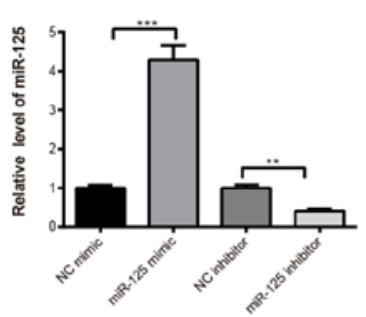

D

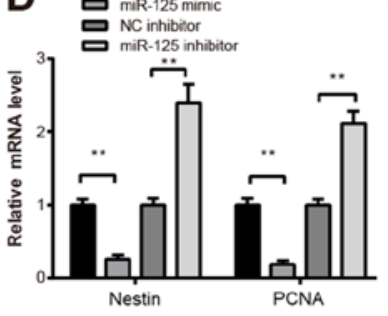

$\mathbf{F}$

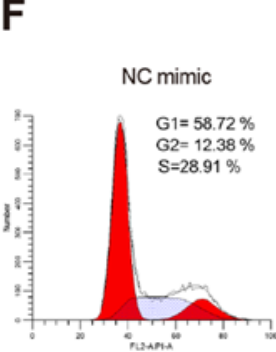

B

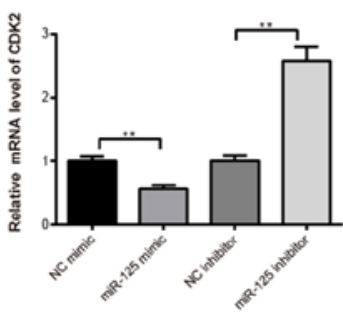

C

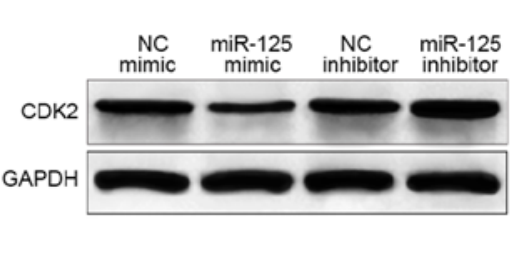

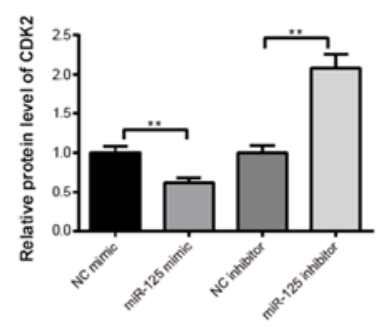

E
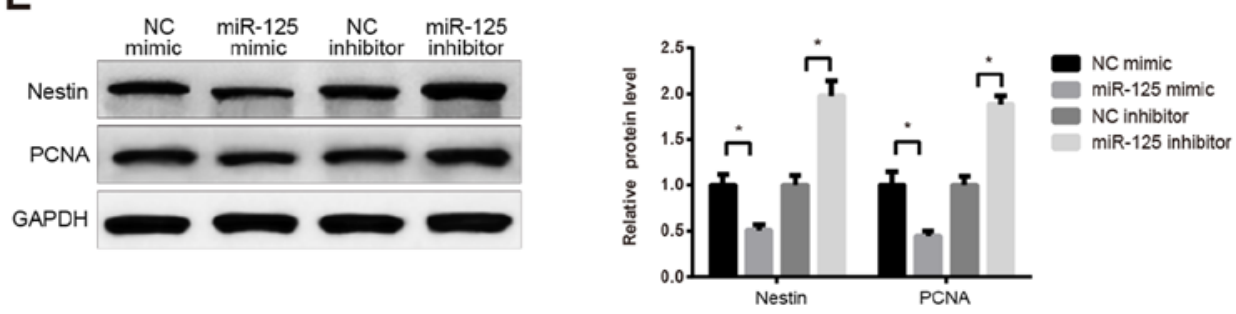
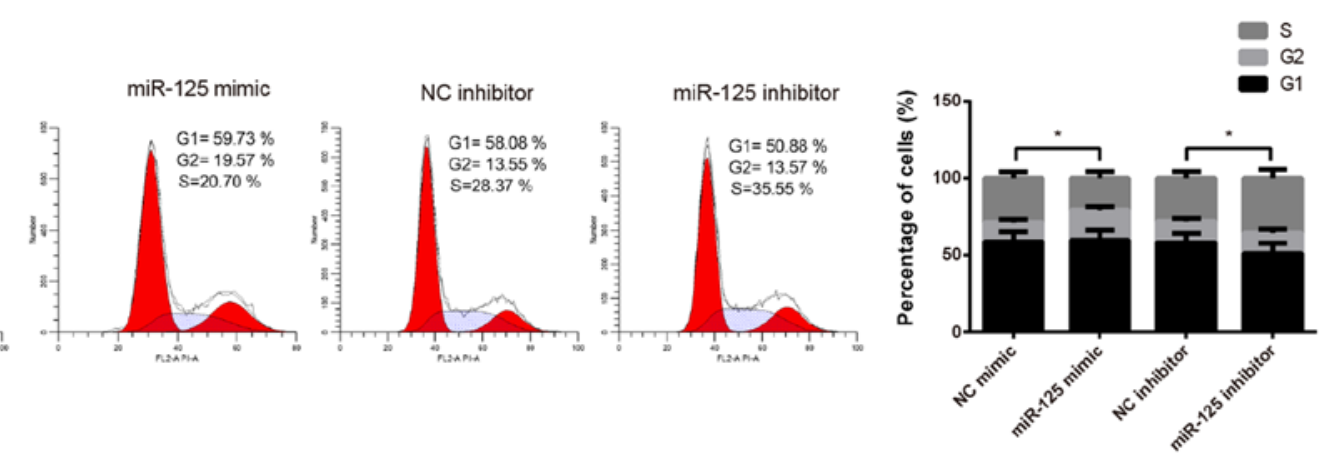

Figure 3. miR-125 inhibited the proliferation of CPCs. Following CPC transfection with miR-125 mimic or miR-125 inhibitor, (A) miR-125 expression and (B) CDK2 expression was examined by RT-qPCR. (C) Protein expression of CDK2 was detected by western blotting. mRNA and protein expression of nestin and PCNA was determined by (D) RT-qPCR and (E) western blotting. (F) Cell cycle analysis was detected by flow cytometry. All data were expressed as the means \pm standard deviation. ${ }^{*} \mathrm{P}<0.05,{ }^{* *} \mathrm{P}<0.01{ }^{* * *} \mathrm{P}<0.001$. PCNA, proliferating cell nuclear antigen; RT-qPCR, reverse transcription quantitative; NC, negative control; CDK2, cyclin-dependent kinase 2; CPCs, cochlear progenitor cells. 
A

wt CDK2 5 '- UUUUCACCCGUGGUCUGACUUGA-3'
| $1|1|||||$
miR-125b-2-3p 3'-UCCAGGGUUCUUGGACUGAACA-5'

mut CDK2 5'- UUUUCACCCGUGGUCACUGAACA-3'
B
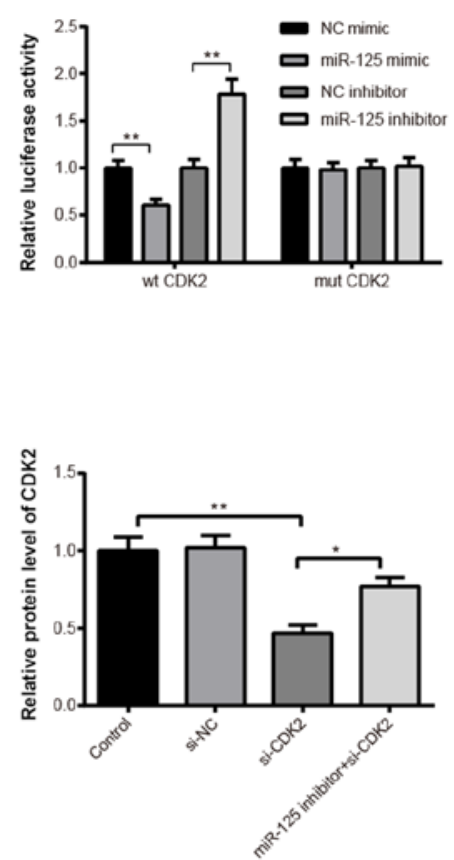

C

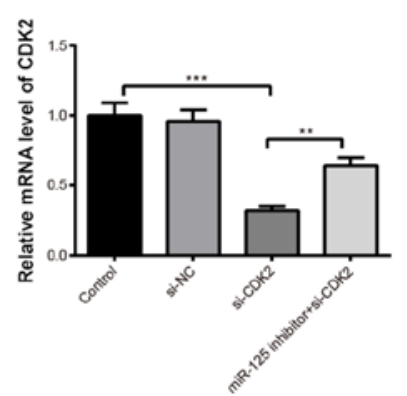

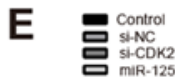

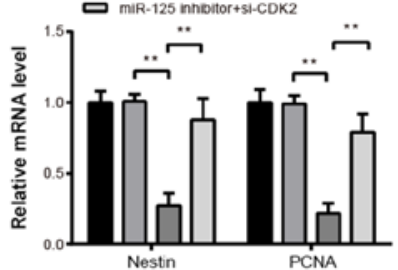

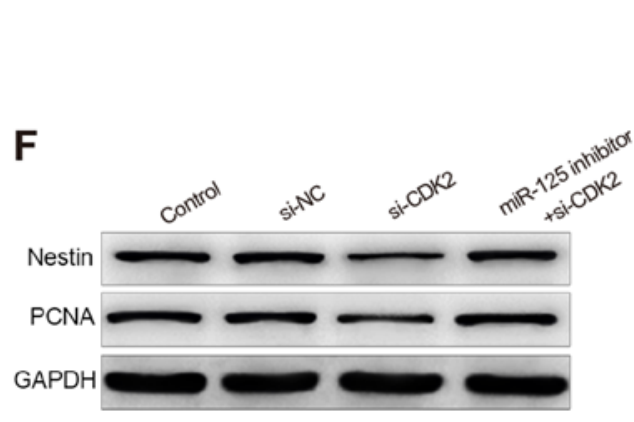
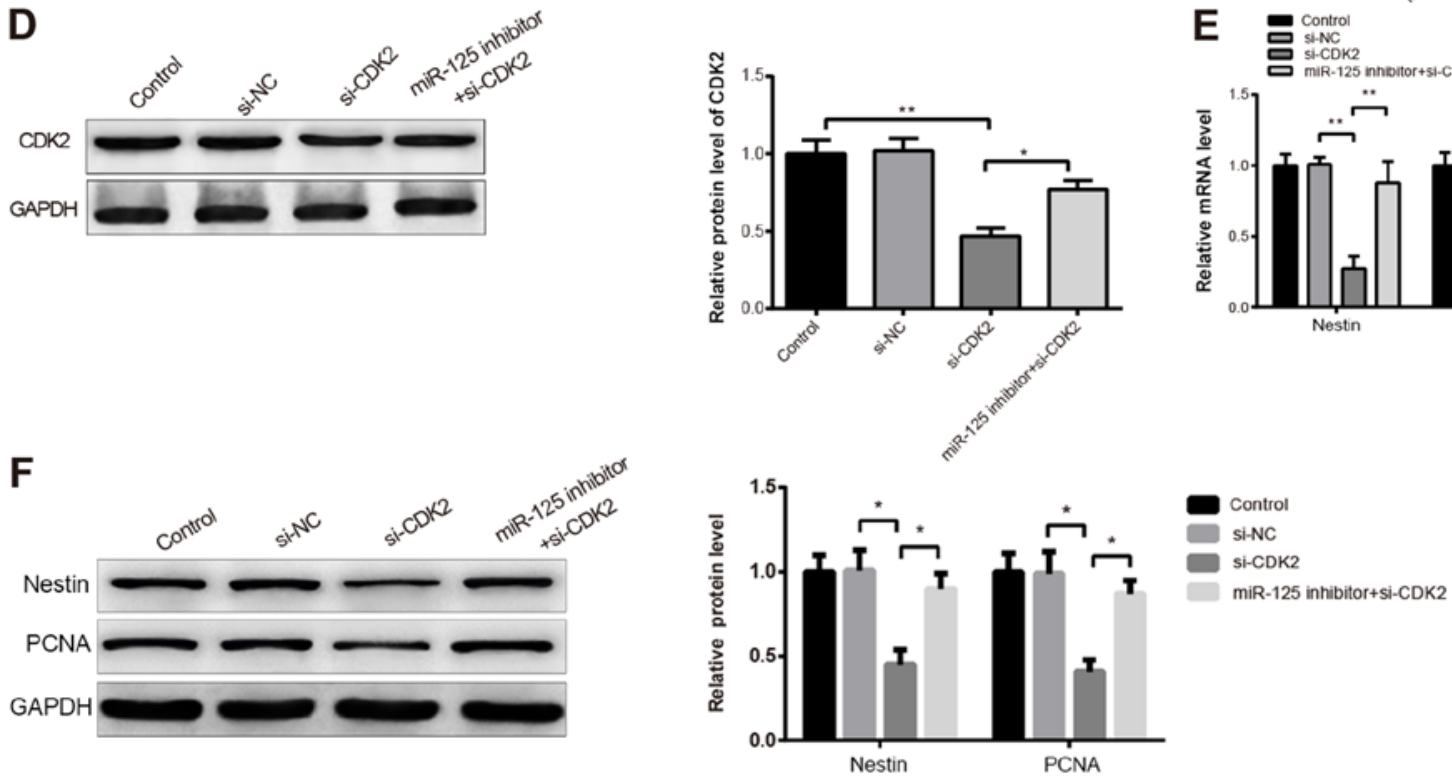

G control
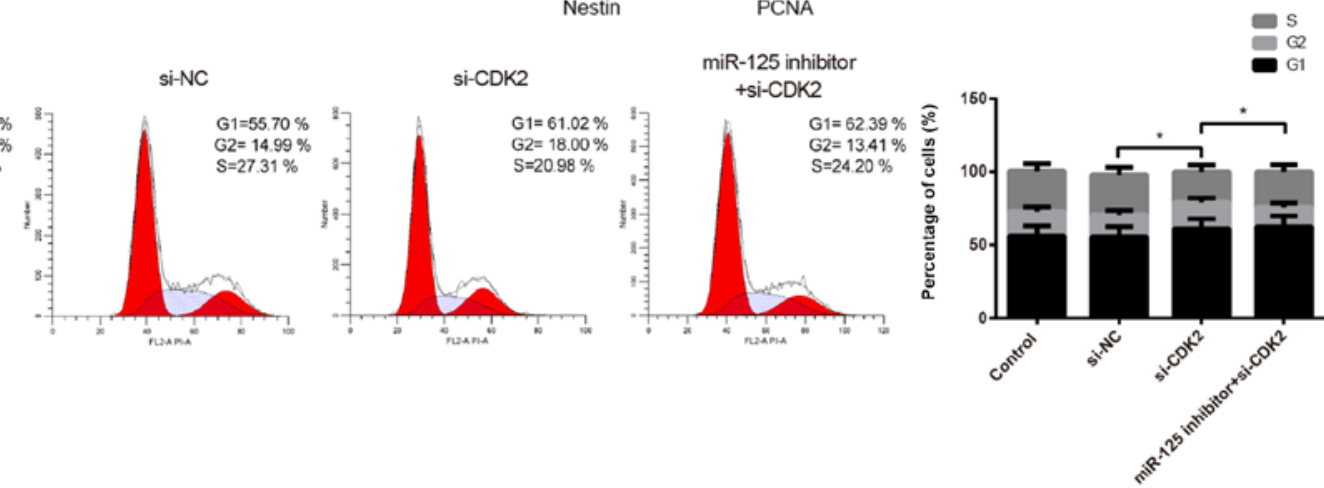

Figure 4. miR-125 regulated CPC proliferation by downregulating CDK2. (A) Following CPC transfection with miR-125 mimic or miR-125 inhibitor, the potential binding sites between miR-125 and CDK2 were predicted. (B) Detection of luciferase activity of 293T cells transfected with wt-CDK2 or mut-CDK2. mRNA and protein expression of CDK2 was determined by (C) RT-qPCR and (D) western blotting in CPCs co-transfected with miR-125 inhibitor and si-CDK2. mRNA and protein expression of nestin and PCNA was measured by (E) RT-qPCR and (F) western blotting. (G) Cell cycle analysis was detected by flow cytometry. All data were expressed as the means \pm standard deviation. ${ }^{*} \mathrm{P}<0.05,{ }^{* *} \mathrm{P}<0.01{ }^{* * *} \mathrm{P}<0.001$. CPCs, cochlear progenitor cells; PCNA, proliferating cell nuclear antigen; RT-qPCR, reverse transcription quantitative; si, small interfering; mut, mutant; wt, wild type; NC, negative control; CDK2, cyclin-dependent kinase 2 .

demonstrated that miR-125 mimic significantly decreased the luciferase activity of $293 \mathrm{~T}$ cells transfected with wt-CDK2 $(\mathrm{P}<0.01)$, and that miR-125 mimic did not alter the luciferase activity of 293T cells transfected with mut-CDK2 compared with the NC mimic group (Fig. 4B). Subsequently, transfection with miR-125 inhibitor significantly increased the luciferase activity of $293 \mathrm{~T}$ cells transfected with wt-CDK2 $(\mathrm{P}<0.01)$, whereas the luciferase activity of $293 \mathrm{~T}$ cells transfected with mut-CDK2 was not changed by the miR-125 inhibitor compared with the NC inhibitor (Fig. 4B).
To test the interactions between miR-125 and CDK2, CPCs cultured for 2 days were co-transfected with miR-125 inhibitor and si-CDK2. The detection of the transfection efficiency of si-CDK2 by RT-qPCR and western blotting revealed a satisfactory CDK2 knockdown in CPCs (Fig. 4C and D; ${ }^{*} \mathrm{P}<0.05$, $\left.{ }^{* *} \mathrm{P}<0.01,{ }^{* * *} \mathrm{P}<0.001\right)$. Furthermore, miR-125 inhibitor upregulated CDK2 expression (Fig. 4C and D; ${ }^{*} \mathrm{P}<0.05,{ }^{* *} \mathrm{P}<0.01$, $\left.{ }^{* * *} \mathrm{P}<0.001\right)$. In addition, the expression levels of nestin and PCNA were significantly decreased following CDK2 knockdown compared with control group (Fig. 4E; $\mathrm{P}<0.01$ ), whereas 
they were significantly increased in the miR-125 inhibitor + si-CDK2 group compared with the si-CDK2 group (Fig. 4E; $\mathrm{P}<0.01)$. Similar results were observed by western blotting (Fig. 4F; $\mathrm{P}<0.05$ ). In addition, the number of cells in the $\mathrm{S}$ phase was decreased in the si-CDK2 group compared with the control group, and was increased in the miR-125 inhibitor + si-CDK2 group compared with the si-CDK2 group (Fig. 4G; $\mathrm{P}<0.05)$. Taken together, these findings suggested that miR-125 may inhibit CPC proliferation by downregulating CDK2.

\section{Discussion}

CPCs have been considered as the best candidates for hair cell regeneration (16). In the past decade, much attention has been given to the regeneration of hair cells from CPCs to rescue hearing loss. For example, a previous study isolated progenitors with high Lgr6 expression levels from transgenic mice and found that high Lgr6 expression elevated the population of progenitor cells, increasing therefore hair cell generation (17). The present study described the role and mechanism of miR-125 in CPC proliferation. Exploring the mechanisms underlying CPC proliferation may account for the treatment of hearing loss following hair cell damage.

To determine the mechanism of CPC differentiation into hair cells, cells were isolated from the cochleae of neonatal rats and further examined. BrdU, a thymine analogue, can be incorporated into new DNA during DNA synthesis (S phase) (18), suggesting that BrdU-expressing cells have proliferation ability. Nestin is a class VI intermediate filament protein associated with pluripotency in pluripotent stem cells, which is abundantly expressed in the developing central nervous system and downregulated in proliferative areas of the dentate gyrus and subventricular zone in adults (19). Nestin-expressing cells present a stem- or progenitor-like character. In the present study, cells isolated from the cochleae of neonatal rats were characterized by observing the number of cells positive for BrdU and nestin. The results demonstrated that nestin was mainly expressed in the cytoplasm whereas BrdU was expressed in the nuclei. Further results suggested that isolated cells had the potential to differentiate into hair cells, as evidenced by the expression of myosin VIIA, which normally functions in the cochlear hair cells of the inner ear. Furthermore, the results from the present study demonstrated that CPC proliferation gradually decreased in a time-dependent manner.

miRNAs have integral roles in regulating cell proliferation, differentiation and maturation in addition to the cell fate determination of stem cells (20). Numerous miRNAs, such as miR-124 and miR-182, have been implicated in inner ear development and hair cell fate by modulating downstream mRNA expression $(21,22)$. However, the roles of miRNAs in mediating CPC proliferation remain unclear. The inhibitory effects of miR-125 family members on cell proliferation have been reported in various types of cell, including colorectal cancer cells, cardiomyocytes and osteoblasts (23-25). In the present study, miR-125 was found to be downregulated and eventually upregulated in the progenitor spheres. Furthermore, overexpression of miR-125 in CPCs decreased the levels of nestin and PCNA as well as the number of cells in the $\mathrm{S}$ phase. A previous study demonstrated that NEUROG1 overexpression can inhibit the proliferation of otic progenitors by decreasing CDK2 expression (26). In the present study, the expression of CDK2 was increased and then decreased in CPCs, suggesting the involvement of CDK 2 in CPC proliferation. In addition, miR-125 upregulation decreased the expression of CDK2 and CDK2 expression was upregulated by the introduction of miR-125 inhibition into CPCs. To determine the mechanism of miR-125 on CPC proliferation, the relationship between miR-125 and CDK2 was investigated. A dual luciferase reporter assay demonstrated that miR-125 could negatively target CDK2. The results also revealed that miR-125 inhibition reversed the suppressive effect of CDK2 knockdown on CPC proliferation. Taken together, this study demonstrated that miR-125 inhibited CPC proliferation by downregulating CDK2. This study did not investigate the role of the miR-125/CDK2 axis in other biological processes of CPCs, such as differentiation, although results confirmed that isolated CPCs had the potential to differentiate into hair cells and that CPC proliferation was attenuated after incubation for 7 days. Previous studies have reported the essential roles of miR-125 and CDK2 in progenitor or stem cell differentiation (27-29). The function of miR-125/CDK2 axis in CPC differentiation requires further investigation.

In summary, the results from the present study suggested that CPCs may have the potential to differentiate into hair cells. In addition, miR-125 inhibited CPC proliferation by negatively targeting CDK2. Hearing loss treatment based on progenitor or stem cell strategies could therefore be considered; however, the mechanism of CPC proliferation needs to be further investigated. This study attempted to explain the molecular mechanisms of miRNA-125 on the regeneration of hair cells.

\section{Acknowledgements}

Not applicable.

\section{Funding}

This work was supported by the National Natural Science Foundation of China for Distinguished Young Scholars (grant no. 81800921), Natural Science Foundation of Hunan Province (grant no. 2020JJ5303), the Foundation of Health and Family Planning Commission of Hunan Province (grant no. C2017033) and Hunan Provincial Technology Innovation-Oriented Projects (grant no. 2018SK50705).

\section{Availability of data and materials}

The datasets used and/or analysed during the current study are available from the corresponding author on reasonable request.

\section{Authors' contributions}

TP conceptualized and designed the experiments and supervised the study. JJP performed the experiments. GYM, ZQT, BL and EZ analyzed the data. BL and EZ revised the manuscript critically for important intellectual content. TP and JJP wrote the manuscript. All authors read and approved the final manuscript. 


\section{Ethics approval and consent to participate}

Animal experiments were approved by the Ethics Committee of Hunan Provincial People's Hospital (approval no. 2019S47).

\section{Patient consent for publication}

Not applicable.

\section{Competing interests}

The authors declare that they have no competing interests.

\section{References}

1. Cunningham LL and Tucci DL: Hearing loss in adults. N Engl J Med 377: 2465-2473, 2017.

2. Kim YR, Baek JI, Kim SH, Kim MA, Lee B, Ryu N, Kim KH, Choi DG, Kim HM, Murphy MP, et al: Therapeutic potential of the mitochondria-targeted antioxidant MitoQ in mitochondrial-ROS induced sensorineural hearing loss caused by Idh2 deficiency. Redox Biol 20: 544-555, 2019.

3. Tang M, Yan X, Tang Q, Guo R, Da P and Li D: Potential application of electrical stimulation in stem cell-based treatment against hearing loss. Neural Plast 2018: 9506387, 2018.

4. Lee MY and Park YH: Potential of gene and cell therapy for inner ear hair cells. Biomed Res Int 2018: 8137614, 2018.

5. Roccio M, Perny M, Ealy M, Widmer HR, Heller S and Senn P: Molecular characterization and prospective isolation of human fetal cochlear hair cell progenitors. Nat Commun 9: 4027, 2018.

6. Lin SCY, Thorne PR, Housley GD and Vlajkovic SM: Purinergic signaling and aminoglycoside ototoxicity: The opposing roles of P1 (Adenosine) and P2 (ATP) receptors on cochlear hair cell survival. Front Cell Neurosci 13: 207, 2019.

7. Revuelta M, Santaolalla F, Arteaga O, Alvarez A, Sanchez-Del-Rey A and Hilario E: Recent advances in cochlear hair cell regeneration-A promising opportunity for the treatment of age-related hearing loss. Ageing Res Rev 36: 149-155, 2017.

8. Youm I and Li W: Cochlear hair cell regeneration: An emerging opportunity to cure noise-induced sensorineural hearing loss. Drug Discov Today 23: 1564-1569, 2018.

9. Huh SH, Warchol ME and Ornitz DM: Cochlear progenitor number is controlled through mesenchymal FGF receptor signaling. Elife 4: e05921, 2015.

10. Mittal R, Liu G, Polineni SP, Bencie N, Yan D and Liu XZ: Role of microRNAs in inner ear development and hearing loss. Gene 686: 49-55, 2019.

11. Pang J, Xiong H, Yang H, Ou Y, Xu Y, Huang Q, Lai L, Chen S, Zhang Z, Cai Y and Zheng Y: Circulating miR-34a levels correlate with age-related hearing loss in mice and humans. Exp Gerontol 76: 58-67, 2016

12. La Torre A, Georgi S and Reh TA: Conserved microRNA pathway regulates developmental timing of retinal neurogenesis. Proc Natl Acad Sci USA 110: E2362-E2370, 2013.

13. Li L, Wang Q, Yuan Z, Chen A, Liu Z, Wang Z and Li H: LncRNA-MALAT1 promotes CPC proliferation and migration in hypoxia by up-regulation of JMJD6 via sponging miR-125. Biochem Biophys Res Commun 499: 711-718, 2018.

14. Spencer SL, Cappell SD, Tsai FC, Overton KW, Wang CL and Meyer T: The proliferation-quiescence decision is controlled by a bifurcation in CDK2 activity at mitotic exit. Cell 155: 369-383, 2013.
15. Livak KJ and Schmittgen TD: Analysis of relative gene expression data using real-time quantitative PCR and the 2(-Delta Delta $\mathrm{C}(\mathrm{T})$ ) method. Methods 25: 402-408, 2001.

16. Song YL, Tian KY, Mi WJ, Ding ZJ, Qiu Y, Chen FQ, Zha DJ and Qiu JH: Decreased expression of TERT correlated with postnatal cochlear development and proliferation reduction of cochlear progenitor cells. Mol Med Rep 17: 6077-6083, 2018.

17. Zhang Y, Guo L, Lu X, Cheng C, Sun S, Li W, Zhao L, Lai C, Zhang S, Yu C, et al: Characterization of Lgr6 ${ }^{+}$cells as an enriched population of hair cell progenitors compared to $\mathrm{Lgr}^{+}$cells for hair cell generation in the neonatal mouse cochlea. Front Mol Neurosci 11: 147, 2018.

18. Gao J, Wan F, Tian M, Li Y, Li Y, Li Q, Zhang J, Wang Y, Huang X, Zhang L and Si Y: Effects of ginsenoside-Rg1 on the proliferation and gliallike directed differentiation of embryonic rat cortical neural stem cells in vitro. Mol Med Rep 16: 8875-8881, 2017.

19. Takeda H, Dondzillo A, Randall JA and Gubbels SP: Challenges in cell-based therapies for the treatment of hearing loss. Trends Neurosci 41: 823-837, 2018.

20. Hei R, Chen J, Qiao L, Li X, Mao X, Qiu J and Qu J: Dynamic changes in microRNA expression during differentiation of rat cochlear progenitor cells in vitro. Int J Pediatr Otorhinolaryngol 75: 1010-1014, 2011

21. Huyghe A, Van den Ackerveken P, Sacheli R, Prevot PP, Thelen N, Renauld J, Thiry M, Delacroix L, Nguyen L and Malgrange B: MicroRNA-124 regulates cell specification in the cochlea through modulation of Sfrp4/5. Cell Rep 13: 31-42, 2015.

22. Wang XR, Zhang XM, Du J and Jiang H: MicroRNA-182 regulates otocyst-derived cell differentiation and targets T-box1 gene. Hear Res 286: 55-63, 2012.

23. Yang M, Tang X, Wang Z, Wu X, Tang D and Wang D: miR-125 inhibits colorectal cancer proliferation and invasion by targeting TAZ. Biosci Rep 39: BSR20190193, 2019.

24. Li L, Zhang M, Chen W, Wang R, Ye Z, Wang Y, Li X and Cai C: LncRNA-HOTAIR inhibition aggravates oxidative stress-induced H9c2 cells injury through suppression of MMP2 by miR-125. Acta Biochim Biophys Sin (Shanghai) 50: 996-1006, 2018.

25. Tu XM, Gu YL and Ren GQ: miR-125a-3p targetedly regulates GIT1 expression to inhibit osteoblastic proliferation and differentiation. Exp Ther Med 12: 4099-4106, 2016.

26. Song Z, Jadali A, Fritzsch B and Kwan KY: NEUROG1 regulates CDK 2 to promote proliferation in Otic progenitors. Stem Cell Reports 9: 1516-1529, 2017.

27. Kaur S, Abu-Shahba AG, Paananen RO, Hongisto $\mathrm{H}$, Hiidenmaa H, Skottman H, Seppanen-Kaijansinkko R and Mannerström B: Small non-coding RNA landscape of extracellular vesicles from human stem cells. Sci Rep 8: 15503, 2018.

28. Malgrange B, Knockaert M, Belachew S, Nguyen L, Moonen G, Meijer L and Lefebvre PP: The inhibition of Cyclin-dependent kinases induces differentiation of supernumerary hair cells and Deiters' cells in the developing organ of Corti. FASEB J 17: 2136-2138, 2003.

29. Shenoy A, Danial M and Blelloch RH: Let-7 and miR-125 cooperate to prime progenitors for astrogliogenesis. EMBO J 34: 1180-1194, 2015.

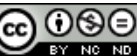

This work is licensed under a Creative Commons Attribution-NonCommercial-NoDerivatives 4.0 International (CC BY-NC-ND 4.0) License. 